\title{
tic\&société
}

Vol. 5, $n^{\circ} 1 \mid 2011$

TIC et genre

\section{Effet de genre : le paradoxe des études d'informatique}

Isabelle Collet

\section{(2) OpenEdition}

Journals

Édition électronique

URL : http://journals.openedition.org/ticetsociete/955

DOI : 10.4000/ticetsociete.955

Éditeur

Association ARTIC

\section{Référence électronique}

Isabelle Collet, «Effet de genre : le paradoxe des études d'informatique », tic\&société [En ligne], Vol. 5, n

- 1 | 2011, mis en ligne le 05 octobre 2011, consulté le 19 avril 2019. URL : http://

journals.openedition.org/ticetsociete/955; DOI : 10.4000/ticetsociete.955 
tic\&société - 5 (1), 2011

\title{
Effet de genre : le paradoxe des études d'informatique
}

\author{
Isabelle COLLET \\ Groupe relations interculturelles et formation des enseignants - genre et \\ éducation (GRIFE-GE) \\ Université de Genève \\ Faculté de Psychologie et des Sciences de l'Education \\ Institut universitaire de formation des enseignants \\ 40, Boulevard du Pont d'Arve \\ $\mathrm{CH}-1205$ Genève \\ isabelle.collet@unige.ch
}

Isabelle Collet est chargée d'enseignement à I'Institut universitaire de formation des enseignants (IUFE), membre du Groupe relations interculturelles et formation des enseignants - genre et éducation (GRIFE-GE) de la Section des sciences de l'éducation de l'Université de Genève. Informaticienne scientifique de formation initiale, elle est docteure en sciences de l'éducation et chercheuse associée à l'équipe "Genre, savoirs et éducation " du Centre de recherche éducation formation (CREF) de l'université Paris-Ouest Nanterre La Défense. Elle a publié en 2006 chez l'Harmattan : "L'informatique a-t-elle un sexe », qui a reçu le prix de l'Académie française des sciences morales et politiques. 


\title{
Effet de genre : le paradoxe des études d'informatique
}

\begin{abstract}
Résumé
Parmi les études scientifiques et techniques, l'évolution de la discipline informatique attire l'attention. En effet, à partir des années 1980, de plus en plus d'hommes choisissent cette filière alors que dans le même temps, le nombre de femmes reste constant. Nous faisons l'hypothèse que le choix d'étude et l'exercice d'une profession sont des pratiques qui sont toujours médiées par un système symbolique. Le choix d'une filière d'études en Sciences et technologie de l'information et de la communication (STIC) ne s'effectue pas sur la réalité mal connue des métiers de ce secteur mais sur les représentations sociales à la disposition du grand public, nourries par l'imaginaire de l'informatique. Avant l'arrivée du micro-ordinateur, les métiers de l'informatique étaient des métiers scientifiques du tertiaire plutôt attractifs pour les jeunes femmes techniciennes ou ingénieurs. Avec l'arrivée du micro-ordinateur, la représentation de l'informaticien, telle qu'on la voit dans la science-fiction, s'est figée sur un homme pris dans une relation exclusive avec l'ordinateur, représentation très éloignée de la réalité des métiers de l'informatique et qui, loin de disparaître avec la multiplication des usages, se renforce.
\end{abstract}

Mots-clé : genre, informatique, enseignement supérieur, science-fiction, stéréotype

\begin{abstract}
Among the technical and scientific studies, the evolution of the computer sciences draws attention. From the 1980s, more and more men have chosen this field but in the same time, the number of women has remained the same. To explain this masculinisation, we have considered several hypotheses. Our hypothesis is : the choice of studies and job is made through a mix between practices and symbolic representations. Before the micro computer apparence, IT jobs were perceived as "office jobs", suitable for women in technical fields. Then, the representation of the computer scientist sticks on a man solely enthralled by his computer. This representation is very far from the reality the everyday job but does not disappear with the huge variety of computer use and purpose.
\end{abstract}


Keywords : gender, informatics, higher education, science fiction, stereotype

\section{Resumen}

Entre de los estudios científicos y técnicos, la evolución de la disciplina informática llama la atención. En efecto, a partir de los años 80 , cada vez más hombres eligen esta opción mientras que, al mismo tiempo, el número de mujeres se mantiene estable. Hacemos la hipótesis que la elección del estudio y el ejercicio de una profesión son prácticas que están siempre mediatizadas por un sistema simbólico. A través de este imaginario, se reinterpreta el género. Así, antes de la llegada del micro-ordenador, los oficios de la informática eran oficios científicos del sector terciario más atractivos para las mujeres técnicas o ingenieras. Con la llegada del microordenador, la representación del informático, tal y como se refleja en la ciencia ficción, es la de un hombre percibido a través de una relación exclusiva con el ordenador, representación muy alejada de la realidad de los oficios de la informática y que, lejos de desaparecer con el aumento de los usos, se renfuerza.
\end{abstract}

Palabras claves : género, informática, enseñanza superior, ciencia ficción, estereotipo 
Effet de genre : le paradoxe des études d'informatique

L'inégal accès des hommes et des femmes aux Sciences et technologies de l'information et de la communication (STIC) a été constaté tant au niveau mondial qu'européen : en 2004, dans les pays de l'Union européenne, les femmes représentaient $22 \%$ de l'ensemble des diplômés en informatique (Gras-Velazquez, Joyce, \& Debry, 2009), 27,8\% des cadres en informatique et système d'information sont des femmes, 9,6\% seulement sont ingénieures système (Catalyst, 2008).

Les disparités restent importantes d'un pays à l'autre. Par exemple, au niveau du doctorat, les 25 pays de l'Union européenne diplôment 346 femmes pour 1517 hommes, (soit environ 18,6\%). Ce pourcentage correspond aussi à celui de la France (76 femmes pour 328 hommes). En revanche, la Belgique ne compte que 2 femmes pour 60 hommes en thèse d'informatique et la Suisse, 4 femmes pour 49 hommes (She Figures, 2006).

Le Bureau international du travail signale que: "Au Royaume-Uni et aux Etats-Unis, les femmes qui étudient les technologies de l'information ne représentent, dans tous les cycles, que $20 \%$ des étudiants en informatique, et ce pourcentage continue à baisser. Le profil des inscrits aux cours de formation de Microsoft, dont $11 \%$ seulement sont des femmes, confirme l'image stéréotypée d'une branche d'activité qui est entre les mains d'hommes jeunes.» (BIT, 2002)

Toutefois, il est difficile d'obtenir des données sexuées internationales sur la population en études d'informatique. Le terme d'informatique pose lui-même problème, à cause de sa polysémie en français et aussi de son absence d'équivalent en anglais.

En France, l'informatique, c'est finalement tout ce qui se fait avec un ordinateur, avec une frontière entre la maîtrise et l'usage de plus en plus diffuse, au point que certains auteurs décident de ne plus parler que d'usage (Hourbette, 2010). En outre, pendant longtemps, la discipline informatique n'a pas été séparée des mathématiques, de l'électronique ou des télécoms dans les statistiques.

Dans les dernières années du XXe siècle, le terme de STIC, a fait son apparition, afin justement de palier ces imprécisions. L'ASTI, la société savante des STIC, a été créé en 1998. Elle indique dans ses statuts que "L'informatique, l'automatique, le traitement du signal, les mathématiques, l'électronique, les télécommunications sont en particulier présentes" au sein des STIC. Les STIC ne sont donc pas l'informatique... Mais, en dehors des spécialistes, qui connait les STIC ? L'Office national d'information sur les enseignements et les 
professions (ONISEP) parle "des métiers de l'informatique et des télécoms" alors que c'est bien des STIC dont il s'agit. Le terme si pratique d'informatique, même s'il est incapable de refléter réellement le contenu des filières d'études STIC, est celui qui est utilisé par le grand public, par les élèves, les enseignante-s et les professionnel-le-s de l'orientation. C'est pourquoi dans l'enquête, nous avons préféré parler d'informatique et d'informaticien-ne, simplement parce que ce sont les temes toujours en usage au sein de la population étudiée.

En France, dans les grandes écoles d'ingénieurs, la fréquentation de la filière informatique suit, en ce qui concerne les femmes, une courbe surprenante (Marry, 2004, p. 109). Alors que dans toutes les filières scientifiques et techniques, la part des femmes augmente régulièrement (de $5 \%$ en moyenne en 1972, à $26 \%$ en 2010), l'informatique est le seul domaine où, après avoir été proportionnellement bien représentée, la part des femmes est en nette régression. En effet, de 1972 à 1985, le pourcentage des femmes en informatique est supérieur au pourcentage moyen des femmes ingénieures, toutes écoles confondues. En 1983, l'informatique est le deuxième secteur ${ }^{1}$ comportant le plus de femmes diplômées $(20,3 \%), 6$ points au dessus de la moyenne des femmes ingénieures ${ }^{2}$. Aujourd'hui, les filières STIC diplôment seulement $11 \%$ de femmes, alors la moyenne toute école confondue est de $25 \%$.

En l'espace de vingt ans, la part des femmes en informatique a été divisée par deux.

\footnotetext{
${ }_{2}^{1}$ Le premier étant l'agro-alimentaire avec $27,2 \%$.

2 Secteurs pris en compte: Ecoles généralistes, Défense Nationale, Aéronautique, Agriculture et Industrie agro-alimentaire, BTP et Mines, Informatique, Electricité et Télécoms, Mécanique, Physique et Chimie, Textiles, Autres.
} 
Effet de genre : le paradoxe des études d'informatique

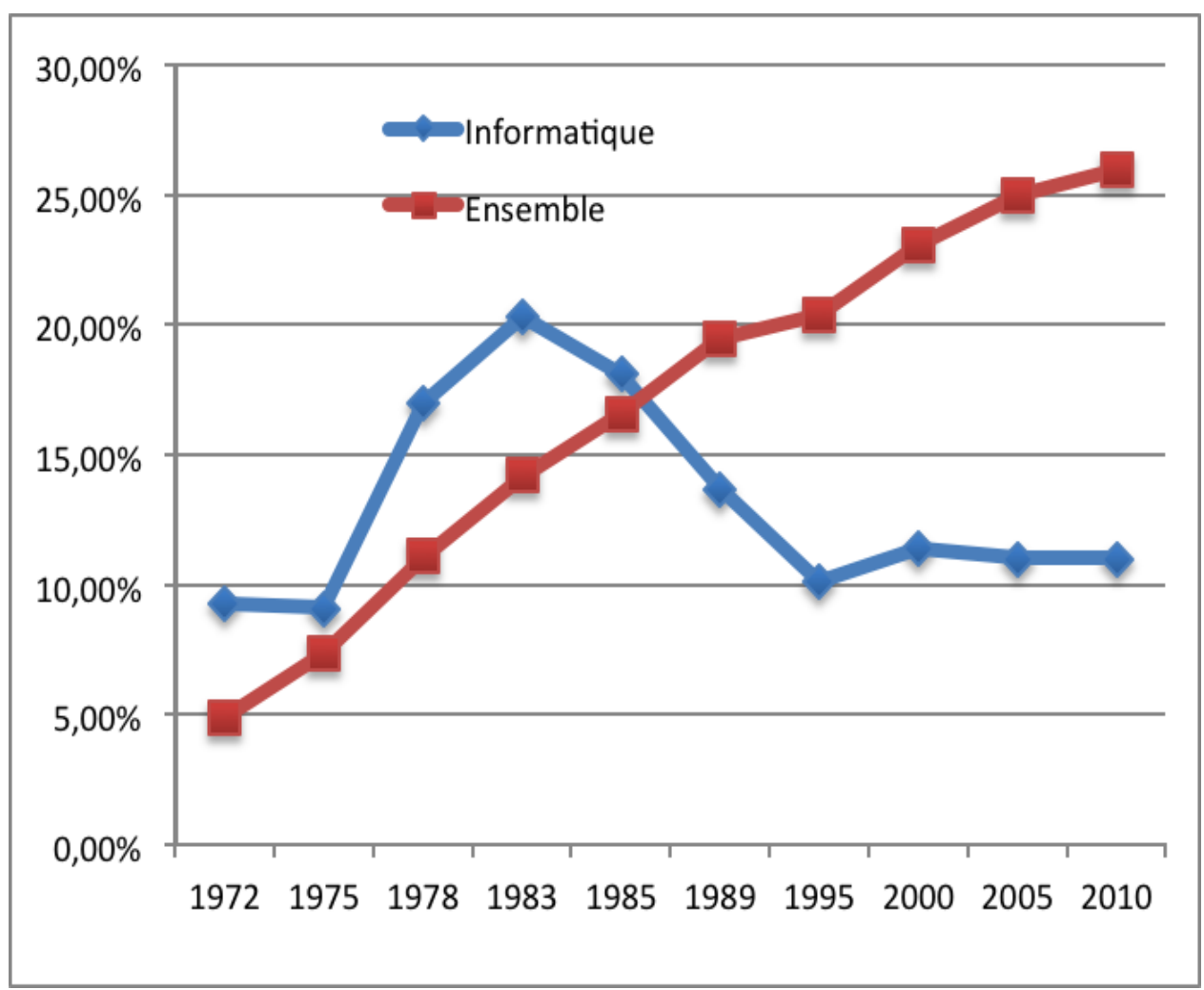

Figure 1 : Pourcentage des femmes diplômées d'écoles d'ingénieurs de 1972 à $2010^{3}$, la rubrique informatique a été renommée STIC au début des années 2000.

Cette situation n'est pas propre à la France, d'autres pays européens constatent également la diminution de la part des femmes parmi les diplômé-e-s en STIC, en particulier l'Allemagne et le Royaume-Uni (Klawe \& Leveson, 1995 ; Oechtering et Behnke, 1995 ; Schinzel, 1997).

3 D'après (Marry, 2004, p.109) sauf 2005 et 2010, chiffres provenant de l'enquête du Conseil national des ingénieurs et des scientifiques de France. 
Regardons maintenant ces chiffres d'une autre manière. Sur la figure suivante, ce ne sont plus les pourcentages qui sont représentés, mais le nombre d'hommes et de femmes diplômés des écoles.

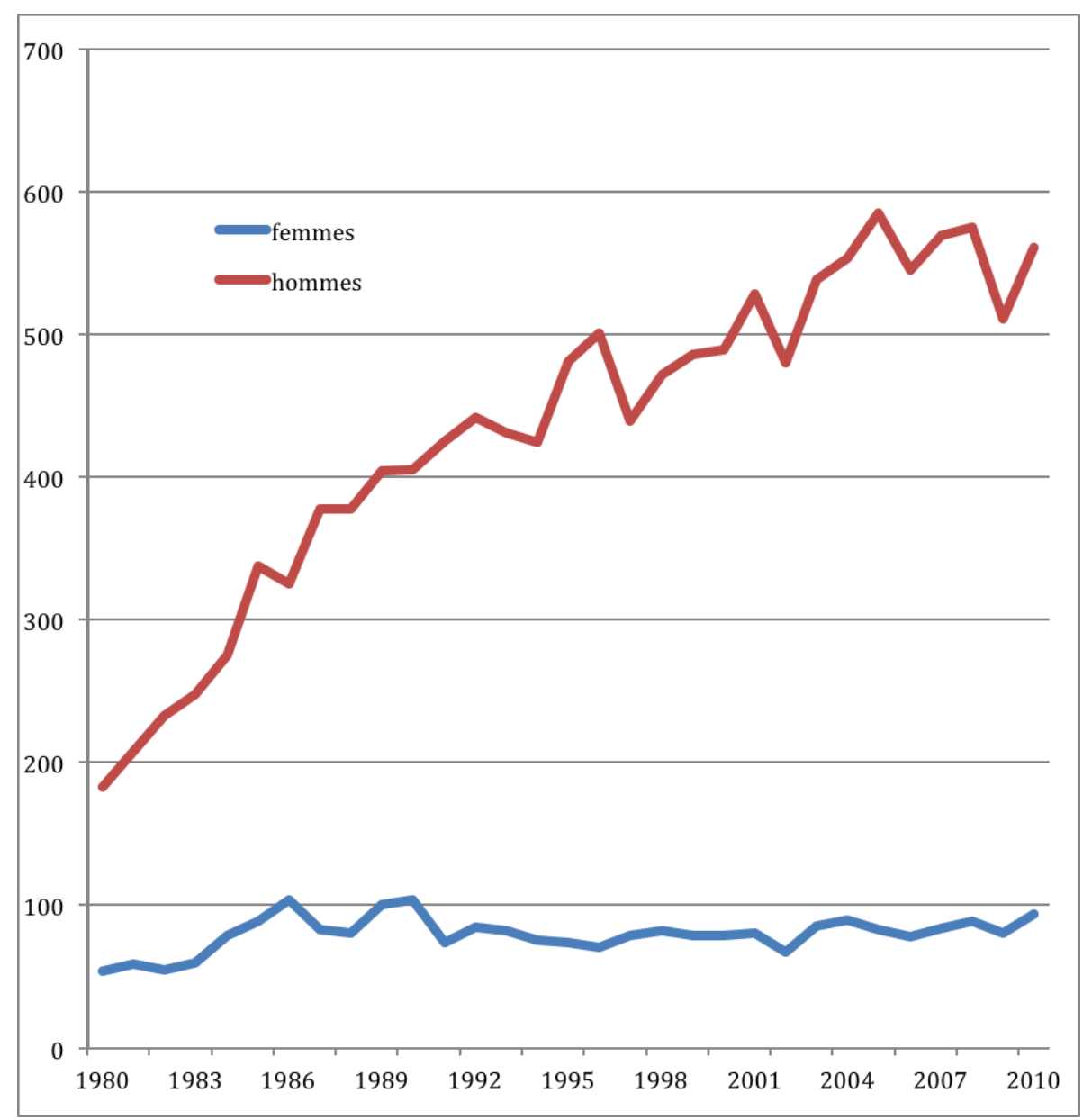

Figure 2 : Nombre d'étudiant-e-s diplômé-e-s de l'option informatique puis STIC dans cinq écoles ${ }^{4}$ d'ingénieurs de 1980 à 2010

\footnotetext{
${ }^{4}$ Les écoles prises en compte sont : les INSA Rennes, Lyon, Toulouse (à partir de 1984), I'ENSIMAG de Grenoble, I'ESIEA de Paris. Données fournies par les scolarités des départements des écoles.
} 
Si le nombre de femmes se dirigeant vers les spécialités informatiques / STIC reste à peu près constant, au fur et à mesure que les filières grossissent ou que de nouvelles spécialités s'ouvrent, le nombre d'hommes augmente considérablement. Les écoles qui avaient l'habitude de voir de nombreuses femmes dans leur effectif voient cette population se diluer car le petit nombre de femmes se répartit au fil des années sur une offre toujours plus vaste. Les écoles qui ouvrent dans les années 1980-1990 n'ont, pour la plupart, jamais connu plus de $10 \%$ ou $15 \%$ de femmes dans leurs effectifs.

Ces données sont corroborées par le Conseil national des ingénieurs et des scientifiques de France (CNISF) : sur la totalité des 116970 femmes ingénieures, la spécialité la plus choisie est l'Agronomie et l'Agroalimentaire à $25 \%$, mais le deuxième choix se porte sur les STIC, à $20,4 \%$. Alors que les STIC sont la première spécialité choisie par $32,7 \%$ des 544130 hommes ingénieurs. Sachant qu'il y a quatre fois plus d'hommes que de femmes ingénieur-e-s, on comprend pourquoi la part des femmes en STIC est si faible.

Les STIC sont aussi le secteur dans lequel on constate la plus forte érosion. En sortie d'école, les femmes sont $15 \%$ à posséder la spécialité informatique, et c'est également le deuxième choix des femmes après l'agroalimentaire. Parmi les ingénieures de moins de 30 ans, elles ne sont plus que $11 \%$.

Pour expliquer ces chiffres, on peut déjà rappeler que les femmes sont sousreprésentées dans toutes les filières et métiers scientifiques et techniques. L'idée, historiquement ancrée, qu'il existerait une incompatibilité fondamentale, de l'ordre de la nature, entre les femmes et les sciences ou entre les femmes et les techniques a été largement étudiée (Mathieu, 1991; Ortner, 1998 ; Tabet, 1998). Les longues constructions sociales de l'image des femmes, d'une part, et de l'image des sciences d'autre part, ont abouti à la constitution d'un stéréotype féminin se composant essentiellement de caractéristiques affectives, rapportant les femmes à leurs rôles d'épouse et de mère: docilité, sensibilité, émotivité, préoccupation des sentiments d'autrui, alors que la description du scientifiquetype s'apparente, pour de nombreux traits, au stéréotype masculin : ambitieux, combatif, audacieux, froid, indépendant, logique, rationnel, obsession de l'objet au détriment de la relation (Bem, 1974 ; Hurtig \& Pichevin, 1986 ; Marro, 1992), que cette description émane d'un homme ou d'une femme.

En outre, nous connaissons depuis Nicole Mosconi l'effet de la répartition socio-sexuée des savoirs, qui décide quels faires et quels savoirs sont légitimes pour chaque individu (Mosconi, 1994), selon son sexe et son origine sociale. Si ces recherches nous permettent de comprendre pourquoi le nombre de femmes en STIC reste relativement bas, elles ne nous permettent pas d'expliquer 
Isabelle COLLET

pourquoi, alors qu'on observe une hausse lente et régulière de la proportion des femmes dans la plupart des filières des écoles d'ingénieurs entre 1980 et aujourd'hui, l'évolution des filières d'informatique va en sens inverse. Il faudrait aussi expliquer l'engagement massif des hommes dans ces filières.

On peut faire l'hypothèse que répartition sexuée et répartition des métiers évoluent de manière concomitante : quand la répartition sexuée d'un métier se modifie, pour rationaliser cette évolution, les représentations du métier changent et ces nouvelles représentations en cours de construction viendront favoriser et renforcer cette modification.

Nous fondons notre hypothèse centrale sur le principe suivant: le choix d'étude et l'exercice d'une profession sont des pratiques qui sont toujours médiées par un système symbolique. Ce système symbolique est un mixte de représentations de la réalité et d'imaginaire. Dans le cas de l'informatique, cet imaginaire a une influence très importante, mais différenciée selon le sexe. A travers cet imaginaire, se rejoue le genre, " un rapport social de pouvoir $d u$ groupe des hommes sur le groupe des femmes, qui institue des normes de sexe différenciatrices et hiérarchisantes, et qui a pour effet de faire des techniques les plus performantes, dont l'informatique fait partie, un monopole (relatif) des hommes » (Collet \& Mosconi, 2010).

Dans une première partie, nous montrerons le rôle de l'imaginaire de l'informatique à travers, d'une part l'analyse d'entretiens et d'autre part, des références à ce type de livres et de films de science-fiction dont on sait qu'ils sont lus par les informaticiens (Clermont \& Lallement, 1998). Puis nous explorerons, à l'aide de questionnaires, l'hypothèse de Huteau (1982) qui met en lien représentation de soi et représentation du métier et nous verrons que l'écart soi-prototype est plus grand pour les étudiantes que pour les étudiants en sciences, alors même qu'ils et elles ont un usage de l'ordinateur. Enfin, nous verrons comment l'imaginaire des années 1980 s'est actualisé dans les représentations d'aujourd'hui.

\section{L'informatique, un travail pour techniciennes du tertiaire}

Des recherches réalisées dans d'autres domaines peuvent nous donner des analogies pour comprendre l'originalité de la filière informatique. Catherine Marry a travaillé sur l'arrivée des femmes dans les écoles d'ingénieurs. Elle a analysé la situation spécifique en France de la chimie, discipline choisie par de 
nombreuses femmes ${ }^{5}$. L'auteur impute l'intérêt des femmes pour la chimie «à une tradition qui date de la fin du XIXe siècle, de formation et d'accueil des femmes dans les métiers industriels liés à la chimie à un niveau inférieur à celui d'ingénieur: celui de techniciennes dans les laboratoires ou assistantes ingénieurs dans les bureaux d'études » (Marry, 2004, p.110). En même temps, tout un discours va tenter de naturaliser le goût des femmes pour la chimie, arguant que cette discipline, concrète et expérimentale, nécessite des qualités d'observation, de patience et d'intuition. Un lien sera fait aussi entre chimie et cuisine : Jules Verne, inaugurant le lycée de filles d'Amiens, déclare le 30 juillet 1893 dans Le Progrès de la Somme, que les cours de chimie leur permettront de confectionner un pot-au-feu (cité par Mariotti, 2000, p.92). II faut dire qu'à cette époque, les lycées de jeunes filles ne préparaient à aucun diplôme et n'avaient aucune finalité professionnelle. II fallait donc trouver une finalité " domestique » aux savoirs acquis à l'école.

Nous disposons également d'une histoire du métier de secrétaire dans laquelle Delphine Gardey montre comment la machine à écrire a transformé le métier de secrétaire, exclusivement occupé par des hommes, en un métier presque exclusivement exercé par des femmes. Assimilée à une machine à coudre (Remington les produisait à partir du même châssis), les machines à écrire étaient supposées se pratiquer comme le piano, autre instrument dit féminin pour la bourgeoisie du XIXe siècle. La pratique des machines à écrire s'est symbolisée dans une représentation qui suppose patience, minutie, attention et précision (Gardey, 2001). Dans une grande entreprise de construction informatique, à l'époque où celle-ci produisait des machines pour la mécanographie, les femmes furent, dès 1933, placées au câblage, " activité de précision pour laquelle l'entreprise [...] faisait appel à leur supposée minutie ou plus exactement à leurs compétences de couturières " (Stevens, 2007, p. 446). Les jeunes filles embauchées étaient titulaires d'un CAP couture. Comme le montre la sociologie du travail (Maruani \& Nicole, 1990), ou l'ethnologie (Tabet, 1998), l'argument naturaliste est toujours convoqué quand on cherche à caractériser le travail des femmes. II s'agit de rapporter à leur nature les qualités qu'on suppose requises pour exercer précisément les métiers qu'on leur assigne.

Ainsi, quand l'ordinateur est né, il s'est inscrit dans la tradition de la machine à écrire. Dans les années 1970, l'ordinateur était perçu comme une machine de bureau et l'informaticien-ne n'était pas toujours un-e ingénieur-e mais aussi

${ }^{5} 52 \%$ de femmes à l'Ecole nationale supérieure de chimie de Paris en 1997, par exemple (Marry, 2004) 
souvent un-e technicien-ne du secteur tertiaire, employé-e dans une banque ou une grande administration (Collet, 2006). Quand une femme était dans une filière scientifique et réfléchissait à son orientation, l'informatique semblait socialement acceptable, s'exerçant dans des lieux professionnels considérés comme plus appropriés pour les femmes que les chantiers ou les industries. Une enquête réalisée en Malaisie par (Lagesen et Mellström, 2004) montre qu'une telle représentation des métiers des TIC existe encore. A la Faculté d'informatique et technologie de l'information de Kuala Lumpur, les responsables de départements ainsi que la Doyenne sont toutes des femmes. A Penang, il y a $65 \%$ d'étudiantes en informatique, 7 professeurs sur 10 sont des femmes, encadrées par une Doyenne. Les raisons invoquées par toutes ces femmes forment un curieux contraste, quand on les compare à ce que nous savons sur le genre des sciences et techniques en Occident. Lagesen et Mellström décrivent une construction au féminin du métier d'informaticien-ne, qui fonctionne sur les oppositions suivantes :

- lourd/léger : l'informatique n'est pas un travail de force,

- dangereux/sûr : la pratique de l'informatique comporte peu de risques physiques,

- propre/sale : l'informatique n'est pas un travail salissant,

- intérieur/extérieur : l'informatique est un travail de bureau, permettant même de travailler de chez soi.

On peut supposer qu'une perception analogue était à l'œuvre en France jusqu'au début des années 1980. A cette période, deux phénomènes se produisirent simultanément.

Le premier se situe du côté du marché du travail. Les emplois peu qualifiés liés à la production matérielle mais aussi à la saisie et à l'exploitation de données (perforateur-trice, opérateur-trice de saisie...) furent sous-traités, disparurent (Stevens, 2007) ou furent délocalisés à l'étranger. Si des femmes et des hommes diplômés furent embauchés par les entreprises d'informatique, (environ $20 \%$ de femmes en 2002 selon la DARES ${ }^{6}$ ), la division du travail suit la hiérarchie des emplois: ainsi, en France, selon l'étude de l'Observatoire Paritaire des Métiers de l'Informatique, de l'Ingénierie, des Etudes et du Conseil, $(\mathrm{OPIIEC})^{7}$ sur des chiffres de 2005 , les femmes n'étaient respectivement que

${ }^{6}$ Direction de l'animation de la recherche, des études et des statistiques. http://www.travail-emploisante.gouv.fr/etudes-recherche-statistiques-de,76/statistiques,78/metiers-et-

qualifications,83/portraits-detailles-des-metiers-en, $\quad$ 233/h-ingenieurs-et-cadres-de-l,337/h0ingenieurs-et-cadres-techniques, $2350 . \mathrm{html}$

${ }^{7}$ http://www.cicf.fr/cicf-social/cicf-opiiec 
$21 \%$ et $23 \%$ dans les métiers du conseil et du logiciel. Or, en effectif réel, ces métiers sont ceux qui proposent le plus d'emplois : un peu plus de 150000 dans le conseil, et environ 125000 dans le logiciel. Si les femmes sont respectivement $33 \%$ et $36 \%$ dans les traitements des données et banques de données, cela correspond seulement pour les deux secteurs à environ 43000 personnes.

Le deuxième phénomène est lié à l'arrivée du micro-ordinateur. II arrive en France au début des années 1980. C'est probablement en 1981, avec le ZX-81 de Sinclair, qui coûtait environ $1000 \mathrm{~F}$, qu'il commence à se répandre chez les adolescents. II existait déjà des machines plus performantes mais plus chères, telles que l'Apple II, sorties trois ans plus tôt mais leur prix les limitait plutôt aux adultes qu'on appellera plus tard des "geeks ${ }^{8}$. La société Tangerine rencontre un vrai succès en 1984 avec l'ORIC Atmos (successeur déjà de I'ORIC 1) et ATARI s'impose l'année suivante avec ses jeux vidéo. Comme l'ont constaté Josiane Jouët et Dominique Pasquier dans leur étude sur les jeunes et l'audiovisuel, à cette époque, comme au moment de leur étude, les garçons sont équipés les premiers d'objets technologiques, que ce soit le baladeur, la console de jeu ou l'ordinateur (Jouët et Pasquier, 1999). Autour de ces microordinateurs, se sont constitués des petits groupes d'adolescents technophiles, hostiles aux filles, à un âge où les enjeux identitaires les poussent à se positionner en tant que garçons masculins face au groupe " autre », celui des filles (Vouillot, 1999). Dix ans plus tard, au début des années 1990, ces adolescents devenus étudiants débutent leurs études supérieures. Quelle représentation de l'ordinateur avaient-ils ?

\section{L'imaginaire de l'informatique}

Dans le cadre d'un doctorat (Collet, 2006), nous avons interviewé dix-sept informaticien-e-s, diplômés de l'enseignement supérieur' ${ }^{9}$. Parmi ces personnes, quatre hommes sont nés autour de 1970 et ont vu l'arrivée du micro-ordinateur

${ }^{8}$ Fans de gadgets techniques.

${ }^{9}$ Toutes les personnes interviewées avaient en commun les éléments suivants : elles ont fait des études d'informatique dans le cycle initial de leurs études et elles ont exercé un emploi en tant qu'informaticien-ne au niveau cadre. Au total nous avons rencontré 17 personnes, 8 hommes et 9 femmes, âgées de 21 à 48 ans. Sept étaient titulaires d'un bac+5 : quatre ingénieurs (deux hommes et deux femmes) et trois Master (deux femmes). Deux personnes (un homme et une femme) étaient titulaires d'une thèse. Les huit autres personnes possédaient au minimum un diplôme de technienne supérieur-e. 
alors qu'ils étaient encore au collège. Tous les quatre vont nous dire que l'ordinateur leur semblait être un objet passionnant, alors même qu'ils n'avaient aucune idée de son fonctionnement ou de son utilité.

«Le père de mon meilleur copain, je devais avoir 10 ans, était informaticien chez Cll Honeywell Bull (ton admiratif), c'était fabuleux. II amenait chez lui des grosses carcasses d'ordinateurs... c'était complètement mystérieux. » (Pierre, né en 1970)

"Mon goût de la programmation a commencé longtemps avant mes études d'informaticien: j'étais fasciné par l'Apple II que j'avais croisé chez des amis de mes parents » (Paul, né en 1965)

" J'en rêvais même avant d'en avoir un. C'est bizarre. [...] Mon rêve jusqu'à quinze ans, c'était de travailler dans un bureau avec un ordinateur. Je rêvais de passer des heures et des heures devant un micro alors que je ne savais pas ce que c'était. » (Jacques, né en 1975)

Le quatrième interviewé le qualifiera aussi de "magique " tout en reconnaissant que c'était « un gros machin qui prend bien de la place et qui fait pas grand-chose " (Alain, né en 1971). On ne peut s'empêcher d'être surpris quand on voit à quel point des adolescents des années 80 ont pu désirer une machine dont ils ignoraient l'usage...

Un des interviewés avancera une explication à cette attraction si forte car il a un souvenir très précis :

«Dans les films ça avait l'air bien. C'était l'époque du film Wargames ${ }^{10}$, où il y a un ado qui arrive à pirater le système du Pentagone. II se servait d'un vieil ordinateur, il tapait, c'était toujours sur fond noir, écrit en jaune dessus. Y avait des mots de passe à trouver, ça avait l'air vachement marrant. " (Jacques, né en 1975)

Son intérêt pour l'ordinateur et la représentation qu'il en a, lui vient directement de la science-fiction : l'ordinateur est cet objet fabuleux qui permet de pirater le Pentagone. Parmi les huit hommes interviewés, sept font référence

${ }^{10}$ Wargames : film américain réalisé par John Badham et sorti en 1983. 
Effet de genre : le paradoxe des études d'informatique

à la science-fiction dans leur entretien. Une des femmes porte le prénom d'une héroïne de science-fiction.

Des représentations fantasmatiques de la machine prennent leur source dans les mythes et histoires de créatures artificielles (Breton, 1996 ; Menegaldo, 1998), et se sont actualisées dans la science-fiction (Clermont et Lallement, 1998 ; Collet, 2006 ; Warrick, 1980). Avec le micro-ordinateur, elles vont disposer d'un support pour s'ancrer dans un quotidien.

L'informatique mise en scène dans la science-fiction, mais également telle qu'elle est vécue par ses spécialistes, est un univers de pouvoir, non seulement sur les machines, mais aussi sur la société et les personnes. Ce pouvoir est en partie fantasmatique car les informaticiens imaginent comprendre comment fonctionne l'univers réel en écrivant les règles qui permettent d'en décrire des portions. Mais plus l'ordinateur prend de place dans la société, plus il la contrôle, dans les faits, plus il la modélise, également. Les personnes qui côtoient l'environnement informatique, c'est-à-dire pratiquement nous tous et toutes, doivent se soumettre aux règles des informaticiens sous peine de se voir refuser l'accès du monde moderne. Dès lors, avec l'ordinateur, on a l'impression de passer de l'autre côté du miroir. Dans l'univers de l'ordinateur, l'informaticien peut jouer à être tout-puissant ${ }^{11}$.

Patrick Flichy cite Alan Kay, chercheur à Xerox Parc, un des créateurs du micro-ordinateur : "L'informatique met la science à l'envers. Dans la science normale, on vous donne un monde et votre travail est de trouver les règles qui s'appliquent. Dans l'informatique, vous donnez les règles à l'ordinateur et il crée le monde. » (Flichy, 2001, p. 81)

Or, il faut reconnaître que les activités de démiurge se conjuguent rarement au féminin. Dans le monde réel, les femmes sont supposées se contenter de suivre les lois des hommes et de les transmettre. Si on peut se demander quelles sont les similitudes entre l'univers réel et l'univers virtuel, on doit en reconnaître au moins une: les femmes n'écrivent les règles ni de l'un, ni de l'autre.

Un informaticien interviewé nous rapportera les propos d'un de ses formateurs :

\footnotetext{
11 II est bon de rappeler l'étymologie du mot: ordinateur. En 1955, Jacques Perret, professeur de philologie, est sollicité par IBM pour trouver un mot français pour désigner ses machines. II répond : "Que diriez-vous d'ordinateur? C'est un mot correctement formé qui se trouve même dans le Littré, comme adjectif désignant Dieu qui met de l'ordre dans le monde. „ Un fac-similé de la lettre originale est disponible auprès de J.-C. Vey, Direction de la Communication IBM France Tours Septentrion Paris la Défense. Une copie se trouve à la Cité des sciences et de l'industrie à Paris.
} 
« Bon, maintenant qu'on est entre nous, on peut dire la vérité. Alors, deux choses : d'abord, on a gagné, on est les maîtres du monde; on est partout, et nos programmes contrôlent tout. Et ensuite, maintenant qu'on contrôle tout, il va falloir commencer à écrire des programmes qui marchent... " (Paul, né en 1965)

Ces propos, lancés sur le ton de l'humour et de la connivence à un groupe de programmeurs, auraient été pris au pied de la lettre par Jacques, quand il était jeune :

"L'ordinateur pour moi était capable de tout faire. Quand je voyais [...] Wargames, le mec pouvait tout faire devant son ordinateur. [...] L'ordinateur c'était l'outil qu'il fallait avoir, c'était le pouvoir ou plutôt la puissance. » (Jacques, né en 1975)

Presque trente ans se sont écoulés depuis l'arrivée du micro-ordinateur. Cet appareil, souvent présenté alors comme fabuleux ou inquiétant est maintenant posé sur tous les bureaux. Les enfants n'ont plus besoin de rêver que leurs parents leur offrent un ordinateur : il est déjà présent pour peu que le contexte financier le permette. En ce qui concerne l'usage de l'ordinateur et en particulier d'Internet, la fracture numérique n'existe pas entre les garçons et les filles, à partir du moment où l'accès est possible (Vendramin et Valenduc, 2003). Mais du côté de la maîtrise des compétences informatiques et des représentations des métiers des STIC, nous allons voir que rien n'a changé.

\section{Un prototype d'informaticien chez les étudiant-e-s}

Nous avons émis l'hypothèse qu'il existe encore aujourd'hui pour les étudiant-e-s en science un prototype de l'informaticien dont les caractéristiques principales seraient : être un homme, peu sociable, logique, passionné par la technique, plus à l'aise avec les machines qu'avec les humains... C'est-à-dire un prototype directement issu des représentations de l'informatique au moment de l'arrivée du micro-ordinateur et toujours prégnant, même si la réalité du métier, nous le verrons par la suite, ne correspond pas.à ses aspirations. Pour tester cette hypothèse, une enquête par questionnaires a été menée en 2004 
Effet de genre : le paradoxe des études d'informatique

auprès de 363 étudiant-e-s de L1 sciences (toutes disciplines confondues) de l'université Claude Bernard de Lyon ${ }^{12}$.

Les questionnaires comportaient une série de 60 items, constitués soit de traits de personnalité, soit de pratiques sociales ou culturelles par lesquels les étudiant-e-s étaient invité-e-s à définir un-e informaticien-ne. La sélection des adjectifs et des descripteurs liés aux styles de vie et aux valeurs et intérêts professionnels a été empruntée à Cendrine Marro et Françoise Vouillot (1991) qui avaient déjà proposé un questionnaire permettant à des élèves du secondaire de définir le ou la scientifique. Quelques questions ouvertes complétaient le questionnaire, demandant aux étudiants ce qui les attirerait ou les repousserait dans les métiers de l'informatique ${ }^{13}$.

Si on regarde quantitativement les réponses fournies par les étudiant-e-s à la série d'items, on obtient de la part des hommes et femmes une image des métiers de l'informatique fortement stéréotypée. Pour $80 \%$ d'entre eux, un informaticien est bon en technique et en mathématiques, il lit des livres techniques, il est modélisateur, travailleur et persévérant. Les étudiantes sont encore plus précises : pour $80 \%$ d'entre elles, elles ajoutent qu'un informaticien est peu émotif, ne s'intéresse pas à son aspect physique et vestimentaire, ne fait pas de sport, est ambitieux et cherche à avoir des revenus élevés.

Si cette image stéréotypée de l'informatique peut coïncider en grande partie avec le stéréotype de la masculinité hégémonique (Connel, 2005), elle s'apparie mal avec les valeurs supposées féminines. Si on suppose avec Huteau (1982, p. 107) que "l'expression des préférences professionnelles est essentiellement considérée comme le résultat d'une activité de comparaison effectuée par l'individu entre la représentation qu'il a de lui-même et celle qu'il se fait du monde professionnel, on est en droit de penser qu'il est plus difficile pour les étudiantes que pour les étudiants de s'imaginer dans la peau d'un-e futur-e informaticien-ne, comme l'ont montré Marro et Vouillot, (1991) dans le cas des scientifiques.

\footnotetext{
12 Cette enquête a concerné des étudiant-e-s de première année de licence Sciences et Technologies de l'université Claude Bernard - Lyon I, réparti-e-s dans 3 dominantes: L1: Mathématiques - Informatique, L2 : Chimie - Physique - Sciences pour l'ingénieur, L3 : Biochimie Biologie - Sciences de la terre. Dans notre échantillon, les femmes se répartissaient comme suit : $18 \%$ en $L 1,16 \%$ en $L 2,66 \%$ en $L 3$. Les garçons se répartissaient comme suit ; $36 \%$ en $L 1,32 \%$ en L2, $32 \%$ en L3. L'écart entre la répartition des hommes et des femmes dans l'échantillon et dans la population source a été jugé non significatif.

13 Pour plus de précisions sur la constitution du questionnaire et le dépouillement de l'enquête, voir (Collet, 2006).
} 
Quand on analyse qualitativement cette fois les réponses des étudiant-e-s aux questions ouvertes, nous obtenons la représentation suivante: l'informaticien reste assis derrière un ordinateur toute la journée, à faire des choses répétitives et monotones sur des machines sans voir personne. Les problèmes d'articulation vie domestique/vie professionnelle, cités uniquement par un homme et une femme, semblent absents des considérations des étudiant-e-s par rapport aux autres composantes qu'ils et elles imaginent dans les métiers de l'informatique. Nous constatons qu'être informaticien, c'est d'abord "créer des programmes ", alors que le développement de logiciel est une activité qui devient marginale en informatique, représentant moins de $20 \%$ de l'activité.

Cette image, que l'on retrouve dans des études québécoises (Gemme, 2002 ; Solar \& Lafortune, 2003) correspond à la vision de l'informaticien asocial qui programme toute la journée. II existe, certes, mais il est rare et bien peu représentatif des informaticiens en entreprise. Dans les questionnaires, deux aspects essentiels des métiers de l'informatique qui sont la communication et le travail d'équipe, disparaissent presque complètement, cités par moins de 10 personnes sur 363. La constante évolution du secteur, la nécessité de veille technique ne sont citées que par une dizaine d'étudiants-e-s. Deux étudiants parlent d'images sur ordinateur. En tout, le mot « internet » n'a été employé que onze fois.

Pourtant, le paysage informatique s'est profondément transformé avec l'arrivée massive d'Internet, et en particulier chez les jeunes. Mais ces transformations des usages n'ont eu pratiquement aucune incidence sur l'image des métiers, comme si les étudiant-e-s ne pouvaient imaginer des métiers de l'informatique en amont de ces usages, ou encore que quels que soient les usages ou les évolutions techniques, le métier d'informaticien reste immuablement lié à la programmation, l'informatique ne se met pas au service des STIC.

\section{Un stéréotype qui se renforce dans l'imaginaire social}

Ce stéréotype de l'informaticien, loin de disparaître devant la diversité des métiers de l'informatique est en train de devenir de plus en plus prégnant dans l'imaginaire social et la presse se fait le relais du stéréotype. Le 6 février 2007, 
le quotidien gratuit "Matin Plus ${ }^{14}$ " titre dans ses pages Emploi : "Des informaticiens très demandés ". L'article, destiné à inciter les jeunes à s'orienter vers ces métiers, est illustré d'une photo sur laquelle on voit un jeune homme en jean et pull, sur une chaise à roulette, un ordinateur portable sur les genoux. La position qu'il adopte pour taper sur le clavier le montre replié sur la machine, presque enroulé autour. A sa droite, on voit dépasser de nombreux câbles. Tout le fond de l'image, parfaitement visible, est occupé par des écrans et unités centrales d'ordinateurs empilés les unes sur les autres.

Aujourd'hui, la très large majorité des informaticien-ne-s viennent au travail en tailleur ou en costume et passent leur journée dans des bureaux paysagers. Le technicien travaillant en salle machine n'est en rien représentatif du quotidien des métiers de l'information et de la communication. Pour autant, cette image, facilement identifiable par le public aujourd'hui, sert de référence aux discours sur l'informatique. Bien des hommes ne se sentent pas attirés par cette représentation erronée et de moins en moins de femmes s'imaginent dans un tel rôle. Beaucoup craignent de devoir passer leur journée avec de tels collègues, dont les centres d'intérêt sont supposés être strictement limités aux machines.

En outre, les termes de " geek », " nerd » et " hacker " ${ }^{15}$ commencent à être connus du grand public, ou en tout cas des jeunes.

Prenons l'exemple de la grosse sortie cinématographique de l'été 2007 : le quatrième volet d'une célèbre série de films d'action: "Die hard 4, retour en enfer $r^{16} "$. Le thème du scénario est resté le même au long des quatre épisodes : des terroristes menacent la sécurité du pays et le personnage joué par Bruce Willis, policier de New-York, va les combattre à grand renfort de cascades, explosions et poursuites. Dans le quatrième opus, l'attaque terroriste qui désorganise le pays est un piratage informatique. Le personnage qui viendra en soutien du héros est un jeune hacker.

II est intéressant de voir le chemin parcouru entre le film "Wargames » de 1983 cité en interview et ce film de 2007. Dans «Wargames », un jeune hacker, croyant pirater des jeux vidéos, entre par mégarde dans l'ordinateur du

\footnotetext{
${ }_{15}^{14}$ Devenu par la suite : Direct Matin.

15 Les hackers étaient à l'origine des informaticiens brillants, passionnés de programmation et de systèmes informatiques. En passant dans le langage courant, il est devenu synonyme de "Craker », c'est-à-dire de pirate informatique. Le terme anglo-saxon de " geek ", qu'on pourrait traduire par " fan de technique » est devenu un terme dont on peut se revendiquer, alors qu'il était péjoratif il y a peu. Enfin, les «nerds " sont les scientifiques asociaux.

16 « Die hard 4 » est un film de Len Wiseman sur un scénario de Mark Bomback et Doug Richardson avec comme acteur principal Bruce Willis. Le premier épisode : "Piège de cristal » est sorti en 1988.
} 
Pentagone et enclenche le processus menant à la $3^{\mathrm{e}}$ guerre mondiale. Dans "Die hard 4 », un jeune hacker, croyant tester le système de sécurité d'une compagnie qui l'emploie, met en place un code qui va permettre de casser les systèmes de sécurité des réseaux financiers des Etats-Unis. Dans les deux cas, à plus de 20 ans d'intervalle, alors que l'informatique a fortement changé, que l'ordinateur est devenu un objet courant, la même figure du hacker est présentée : un jeune homme brillant, un peu hors-la-loi, passionné de jeux et de science-fiction, et qui a le pouvoir de mettre « la civilisation » en danger en toute inconscience ${ }^{17}$.

Un élément scénaristique diffère tout de même de manière importante. Dans Wargames, le danger vient de l'ordinateur du Pentagone qui accède à l'intelligence et menace de provoquer la guerre nucléaire. En 2007, le pouvoir est entre les mains de ceux qui contrôlent la bourse et les flux monétaires: l'ordinateur est plus connu pour gérer l'économie mondiale que la riposte nucléaire. On ne croit plus à l'ordinateur devenant intelligent et autonome, le danger vient de l'informaticien. Le hacker ${ }^{18}$ tel qu'on le fantasme, est toujours là, il est encore plus puissant qu'avant, parfois sympathique, toujours dangereusement inconscient et parfois réellement malfaisant. II est brillant, asocial, misanthrope ou sympathique et il tient plus que jamais le monde dans sa main.

\section{Conclusions}

Nous avons vu comment le micro-ordinateur, en cristallisant les fantasmes de puissance présents dans la science-fiction, a provoqué un changement dans les représentations des métiers de l'informatique. En comparant le stéréotype de l'informaticien-hacker avec la réalité des métiers des STIC d'aujourd'hui, on

\footnotetext{
17 Des séries et sit-coms mettent également les hackers ou les nerds à la mode, pour en citer quelques unes: IT Crowd (sit-com britannique, depuis 2006, 4 saisons déjà réalisées), Big Bang theory (sit-com américaine depuis 2007, 4 saisons déjà réalisées), Numb3rs (série américaine 20052010, 6 saisons), Nerdz (sit-com française depuis 2007, mini-épisode de 4 min 30.4 saisons déjà réalisées), etc.

${ }^{18}$ Récemment, une hackeuse de fiction est devenue célèbre : il s'agit de Lisbeth Salander de la série Millenium, de Stieg Larson, parue en français en 2006. Néanmoins, Lisbeth Salander peut-elle devenir un modèle d'identification positive ? Si elle est excellente aux commandes d'un ordinateur (peut être même trop pour être réaliste), on ne peut oublier les "à-côté » de sa personnalité. Rendue asociale par une enfance violente où elle finit incarcérée dans un hôpital psychiatrique, elle sera mise abusivement sous tutelle. Puis, au cours de l'histoire, elle est battue et violée.
}

tic\&société - 5 (1), 2011 
Effet de genre : le paradoxe des études d'informatique

peut mesurer le décalage entre cet imaginaire et la réalité des métiers dans ce domaine.

Via les médias, le pirate informatique est devenu selon le cas une sorte de Robin des bois d'Internet, volant la musique et les films aux riches sociétés de l'audio-visuel pour la donner aux internautes, un défenseur des libertés divulguant les secrets honteux des états ou des entreprises ${ }^{19}$, un terroriste capable de faire vaciller la sécurité internationale ou un virtuose anonyme, injustement méconnu mais irresponsable. L'image ambiguë de cette minorité visible des passionnés d'informatique attire, fascine ou repousse. Leur virtuosité informatique fait peur, mais en même temps elle impose une certaine admiration ou envie.

Depuis la rentrée 2005, des départements d'informatique d'universités ou d'IUT constatent que non seulement le vivier dans lequel ils recrutent s'amenuise (même si on ne peut pas encore parler de difficultés à remplir les filières) mais que pour la première fois depuis la création de ces formations, certains groupes ne comportent plus une seule femme.

Plutôt que de s'interroger sur un désintérêt global de la part des femmes, il est plus juste de parler d'un engouement et d'une orientation massive de la part des hommes vers ces filières, ce qui marginalisent des femmes déjà rares.

En outre, à l'Université Libre de Bruxelles (ULB), l'enquête Newtonia ${ }^{20}$ a révélé que la filière informatique est à la fois celle dans laquelle on compte le plus de hommes ( $88 \%$ en 2001 contre $60 \%$ dans les autres disciplines) et également celle dans laquelle on constate le plus de décrochages. On peut formuler l'hypothèse que les jeunes hommes n'y trouvent pas la formation qu'ils étaient venus y chercher: les cours d'informatique ne permettent pas à leurs participants de devenir des hackers, que ce mot soit entendu au sens de programmeur passionné ou au sens de pirate informatique.

Brigitte Rozoy, chargée du secteur Mathématiques - Sciences et technique de l'information et la communication à la Direction générale de la recherche et

\footnotetext{
${ }^{19}$ Les dernières péripéties autour de Wikileaks ont renforcé encore ce discours. Wikileak est un site qui divulgue anonymement des documents témoignant d'une réalité sociale et politique, ou militaire, qui serait cachée. Selon son fondateur, Julien Assang, l'objectif est que WikiLeaks devienne "l'organe de renseignements le plus puissant au monde ». Les polémiques autour du site mettent en avant les compétences de hacker (au sens de pirate) de Julien Assang alors que la plupart des données de ce site ont été envoyées au site et non piratées.

${ }^{20}$ Newtonia (2001), programme d'enquête, de réflexion et d'intervention sur l'évolution actuelle de l'accès des jeunes femmes aux études universitaires de sciences et de sciences appliquées à l'ULB, est disponible sur : http://www.ulb.ac.be/newtonia/
} 
Isabelle COLLET

de l'innovation du Ministère de la recherche et de l'enseignement supérieur en France, note que quand les intitulés des formations en informatique comportent les mots "information " ou "communication ", le pourcentage des femmes y est supérieur à $50 \%$, quand ces formations comportent des intitulés fortement connotés "sciences et techniques", la fréquentation des femmes chute à $7 \%$. Pour autant, "il semble que l'attraction soit plus liée à l'image portée qu'aux réels contenus " et pour une large part identique dans les deux types de formation (Rozoy, 2007).

Presque tous les métiers administratifs utilisent l'ordinateur et un-e secrétaire ou un-e comptable qui passent certainement autant de temps qu'un-e informaticien-ne derrière un écran. Beaucoup d'informaticiens utilisent leur ordinateur comme un outil de bureautique. Toutefois "informaticien" ne renvoie toujours qu'à programmeur. Toutes les activités des STIC : la création de site, l'automatisation de systèmes d'informations, la formation technique aux utilisateurs, la maintenance, le déploiement des réseaux, la vente de produits spécialisés, la formation sur ces mêmes produits... qui nécessitent des compétences à la fois techniques et sociales ne font pas partie de "l'informatique » dans l'esprit des étudiant-e-s ni dans celui du grand public.

Peut-être faudra-t-il définitivement abandonner le terme " informatique », à la fois trop chargé et trop vague, pour ne parler que des métiers des Technologie de l'information et de la communication? Ainsi, des femmes et des hommes pourront effectuer un choix éclairé pour les filières des STIC en fonction de la réalité des métiers auxquels elles préparent et non en se laissant leurrer par la prégnance du stéréotype de l'informaticien. Mais simultanément, les entreprises, si elles veulent réellement réaliser une certaine mixité au sein des équipes, ne doivent pas simplement favoriser l'embauche de femmes, mais être également vigilantes au déroulement de leur carrière, afin de donner des modèles d'identification positive à tous les niveaux de management.

\section{Références bibliographiques}

BEM S. L.,1974, « The measurement of psychological androgyny » Journal of Consulting anf Clinical Psychology, n²4, pp. 155-162.

BIT, 2002, Rapport sur l'emploi dans le monde 2001. Vie au travail et économie de l'information, Genève, BIT

BRETON P., 1996, A l'image de l'homme, Paris, Seuil.

CLERMONT P. et J. LALLEMENT., 1998, « Intelligence et réseau dans la science fiction : Représentation de l'ordinateur dans quelques récits 
Effet de genre : le paradoxe des études d'informatique

contemporains » dans Actes du 11ème Colloque Européen en Informatique et Société, Informatisation et anticipations, entre promesses et réalisations, juin, Strasbourg (France), CREIS (centre de coordination pour la recherche et l'enseignement en informatique et société Paris VI-Jussieu) et Groupe d'étude et de recherche sur la science de l'Université Louis Pasteur, pp. 213224.

CATALYST, 2008., Women in Technology: Maximizing Talent, Minimizing Barriers, dernière consultation le 24 mars 2011, http://www.catalyst.org/publication/36/women-in-technology-maximizingtalent-minimizing-barriers

COLLET I., 2006, L'informatique a-t-elle un sexe ?, hackers, mythes et réalités, Paris, L'Harmattan.

COLLET I. et N. MOSCONI, 2010, «Les informaticiennes : De la dominance de classe aux discriminations de sexe ? "Nouvelles questions féministes, vol.19, n², pp. 100-113

CONNELL R. W., 2005, Masculinities, Australia, Allen \& Unwin.

FLICHY P., 2001, L'imaginaire d'internet, Paris, La découverte.

GADREY D., 2001, La dactylographe et l'expéditionnaire. Histoire des employés de bureau 1890-1930, Paris, Belin.

GEMME B., 2002, «Orientations, représentations et projets de femmes étudiant en informatique » Recherche féministe, vol.15, n², pp. 113-134

GRAS-VELAZQUEZ A., JOYCE A. et M. DEBRY, 2009, Women and ICT : Why are girls still not attracted to ict studies and careers ? Bruxelles, European Schoolnet.

HOURBETTE D., 2010, Genre et usages des technologies de l'information et de la communication. Une étude de cas : une école d'ingénieurs en agronomie, Thèse de doctorat en sciences de l'éducation, Université Paris V.

HURTIG M.-C. et M.-F. PICHEVIN, 1986, La différence des sexes. Paris, Tierce.

HUTEAU M., 1982, « Les mécanismes psychologiques de l'évolution des attitudes et des préférences vis-à-vis des activités professionnelles » $L^{\prime}$ 'orientation scolaire et professionnelle, vol.11, $\mathrm{n}^{\circ} 2$, pp. 107-125.

INGARAO M. et I. COLLET, 2003 La place des femmes dans les sociétés de service et d'ingénierie informatique, enrayer la déféminisation du travail 
informatique. Diagnostic et recommandations à partir d'une enquête en SSII, Paris Ministère de l'Emploi et des Affaires Sociales.

JOUËT J. et D. PASQUIER, 1999, « Les jeunes et la culture de l'écran (volet français d'une enquête comparative européenne », Réseaux, n¹7, pp. 9293.

KLAWE M. et N. LEVESON, 1995 « Women in computing, where are we now ? »Communications of the ACM, vol.58, n¹, pp. 29-35.

LAGESEN V. et U. MELLSTRÖM, 2004, « Why is computer science in Malaysia a gender authentic choice for women? Gender and technology in a crosscultural perspective », Gender \& ICT: Strategies of Inclusion, juin, Bruxelles (Belgique)

MARIOTTI F., 2000, Etudes expérimentales des représentations sociales de la science et des métiers scientifiques selon le sexe au collège et au lycée, Thèse de doctorat, Université Paris VII.

MARRO C., 1992, Garçons et filles face à la science, similarités et divergences quant aux variables intervenant dans le choix d'une orientation scientifique chez les deux sexes, Thèse de doctorat, Université Parix V.

MARRO C. et F. VOUILLOT, 1991, «Représentation de soi, représentation du scientifique-type et choix d'une orientation scientifique chez des filles et des garçons de seconde ", L'orientation scolaire et professionnelle, vol. $3, n^{\circ} 20$, pp. 303-323.

MARRY C., 2004, Une révolution respectueuse : Les femmes ingénieurs ?, Paris, Belin.

MARUANI M. et C. NICOLE, 1990, Au labeur des dames métiers masculins emploi féminins, Paris, Syros Alternatives.

MATHIEU N.-C., 1991, L'anatomie politique : Catégorisation et idéologie du sexe, Paris, Edition Côté-femmes.

MENEGALDO G., 1998, « Le monstre court toujours », dans Gilles Menegaldo (dir), Frankenstein, Paris, Autrement.

MOSCONI N., 1994, Femmes et savoir, la société, l'école et la division sexuelle des savoirs, Paris, L'Harmattan.

OECHTERING V. et R. BEHNKE, 1995, « Situation and advancement measures in germany ", Communications of the ACM, vol.58, n¹, pp. 75-82. 
ORTNER S., 1998, «Is female to male as nature is to culture », dans Joan Landes (dir), Feminism: The public and the private, Oxford, Oxford University press, pp. 21-45.

ROZOY B., 2007, « Etat des lieux sur les femmes dans les STIC», Femmes et mathématiques $\mathrm{n}^{\circ} 8$, pp. 29-33.

SCHINZEL B., 1997, "Why has female participation in German informatics decreased ? ", Women, work and computerization: spinning a web from past to future, Actes de la 6e International IFIP-Conference, Bonn (Allemagne).

EUROPEAN COMMUNITY (2006). She Figures. Women and science statistics and indicators, European community.

SOLAR C. et L. LAFORTUNE, 2003, « Moi et la techno, rapport aux technologies de jeunes cégépiennes et cégépiens », dans Louise Lafortune et Claudie Solar (dir), Femmes et maths, sciences et technos Ste Foy, Presses de l'Université du Québec.

STEVENS H., 2007, « Destins professionnels des femmes ingénieurs. Des retournements inattendus », Sociologie du travail, vol.49, nº, pp. 443-463.

TABET P., 1998, La construction sociale de l'inégalité des sexes : Des outils et des corps, Paris, l'Harmattan.

VENDRAMIN P. et G. VALENDUC, 2003, Internet et inégalités - une radiographie de la fracture numérique. Bruxelles, Labor.

VOUILLOT F., 1999, " Orientation, le reflet des rôles de sexes » dans Françoise Vouillot (dir), Filles et garçons à l'école, une égalité à construire, Paris, CNDP, pp. 81-90.

WARRICK P. S., 1980, The cybernetic imagination in science-fiction, Cambridge, MIT Press. 